\title{
Functional identification of genes responsible for the biosynthesis of 1-methoxy-indol-3-ylmethyl- glucosinolate in Brassica rapa ssp. chinensis
}

\author{
Melanie Wiesner, Monika Schreiner and Rita Zrenner
}

\begin{abstract}
Background: Brassica vegetables contain a class of secondary metabolites, the glucosinolates (GS), whose specific degradation products determine the characteristic flavor and smell. While some of the respective degradation products of particular GS are recognized as health promoting substances for humans, recent studies also show evidence that namely the 1-methoxy-indol-3-ylmethyl GS might be deleterious by forming characteristic DNA adducts. Therefore, a deeper knowledge of aspects involved in the biosynthesis of indole GS is crucial to design vegetables with an improved secondary metabolite profile.
\end{abstract}

Results: Initially the leafy Brassica vegetable pak choi (Brassica rapa ssp. chinensis) was established as suitable tool to elicit very high concentrations of 1-methoxy-indol-3-ylmethyl GS by application of methyl jasmonate. Differentially expressed candidate genes were discovered in a comparative microarray analysis using the $2 \times 104 \mathrm{~K}$ format Brassica Array and compared to available gene expression data from the Arabidopsis AtGenExpress effort. Arabidopsis knock out mutants of the respective candidate gene homologs were subjected to a comprehensive examination of their GS profiles and confirmed the exclusive involvement of polypeptide 4 of the cytochrome P450 monooxygenase subfamily CYP81F in 1-methoxy-indol-3-ylmethyl GS biosynthesis. Functional characterization of the two identified isoforms coding for CYP81F4 in the Brassica rapa genome was performed using expression analysis and heterologous complementation of the respective Arabidopsis mutant.

Conclusions: Specific differences discovered in a comparative microarray and glucosinolate profiling analysis enables the functional attribution of Brassica rapa ssp. chinensis genes coding for polypeptide 4 of the cytochrome P450 monooxygenase subfamily CYP81F to their metabolic role in indole glucosinolate biosynthesis. These new identified Brassica genes will enable the development of genetic tools for breeding vegetables with improved GS composition in the near future.

\section{Background}

Glucosinolates (GS) are amino acid-derived nitrogen- and sulphur-containing plant secondary metabolites characteristic for most families of the order Brassicales [1,2]. Altogether there are about 200 known naturally occurring GS structures [3,4], of which various ecotypes of the model organism Arabidopsis thaliana have about 40 [5]. Depending on the amino acid precursor GS could be divided into three groups: (i) aliphatic GS derived from leucine, isoleucine, valine, and methionine; (ii) aromatic GS derived from phenylalanine and tyrosine; and (iii) indole

\footnotetext{
* Correspondence: zrenner@igzev.de

Leibniz-Institute of Vegetable and Ornamental Crops Grossbeeren and Erfurt e.V., Theodor-Echtermeyer-Weg 1, 14979 Grossbeeren, Germany
}

GS derived from tryptophan. The biosynthesis of GS proceeds through three separate phases, the chain elongation of selected precursor amino acids, the formation of the core GS structure, and finally modifications of the side chain. Several genes of the biosynthetic network and key regulators for GS present in Arabidopsis are known $[6,7]$. The formation of the GS core structure is widely elucidated and genes responsible for secondary modifications of aliphatic GS via oxygenations, hydroxylations, alkenylations and benzoylations have been identified [8]. Indole GS can undergo hydroxylations and methoxylations, with CYP81F2 identified as the gene responsible for 4-hydroxylation of indol-3-ylmethyl GS (I3M) in Arabidopsis [9-11] (Figure 1), together with further 


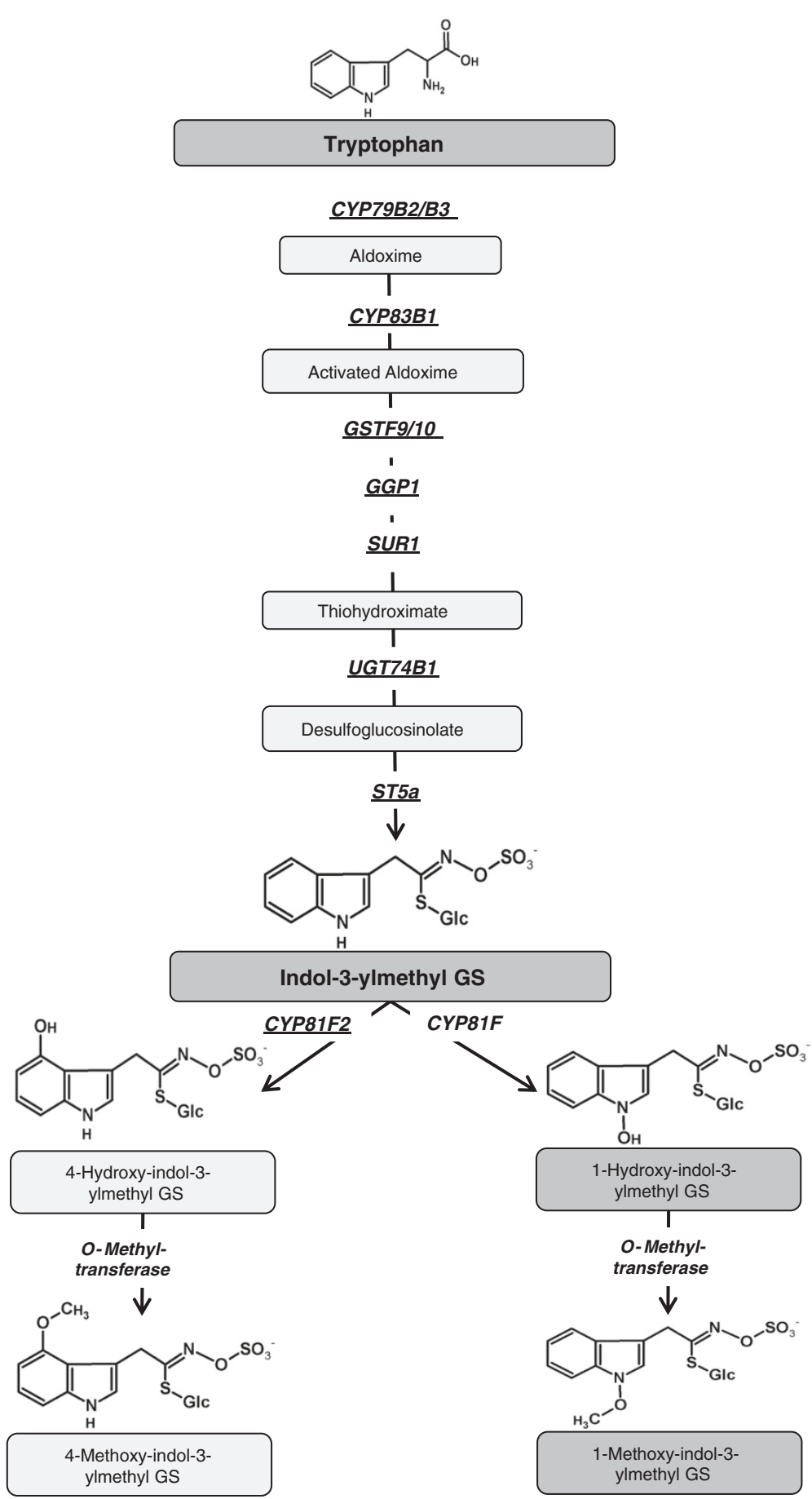

Figure 1 Biosynthesis pathway of indole glucosinolates as known in Arabidopsis thaliana. Enzymes catalyzing each reaction are given with the respective gene name. Identified putative Brassica rapa homologues [14] are indicated with underscores.

members of the CYP81F family of Arabidopsis thaliana as being involved in 4-hydroxylation of indol-3-ylmethyl GS and/or 1-methoxy-indol-3-ylmethyl GS biosynthesis [12]. When tissue is damaged, the thioglucoside linkage of GS is hydrolyzed by myrosinases, enzymes that are spatially separated from GS in intact tissue. In the presence or absence of specifier proteins the degradation results in the formation of a variety of hydrolysis products [13].

The different groups of GS and their various degradation products are extensively studied metabolites. It has been shown that genes encoding enzymes of the 
specific glucosinolate biosynthesis pathways form stable co-expression clusters [15], and group together with tryptophan biosynthetic genes in response to stress conditions [16]. With respect to plant fitness they play important roles in plant defence against herbivores [17] and pathogens [9], and also abiotic stresses like UV-B irradiation specifically changes the GS profile [18]. In addition, there is increasing evidence that evolutionary and ecological forces shape polymorphism at loci involved in the GS-myrosinase defence system [19].

Brassica vegetables are cultivated and consumed worldwide and represent a highly important component in the human diet [20]. Their content of GS is varying dependent on genotype, development and environmental conditions [21] while the composition of GS and their respective degradation products is a major determinant of the characteristic flavor and smell of Brassica vegetables [22]. In addition, the secondary metabolites and their respective degradation products are believed to have protective cancer-preventing activity in higher animals and humans $[23,24]$. However, recent studies also provide evidence that juices of Brassicaceae might also be mutagenic because they form characteristic DNA adducts in bacteria and mammalian cells [25]. It is namely the 1-methoxy-indol-3ylmethyl GS and its degradation products that have been shown to exert these negative effects [26,27].

With this study new genes where identified that are involved in the biosynthesis of indole GS, namely the synthesis of 1-methoxy-indol-3-ylmethyl GS with focus on Brassica vegetables. After establishing the leafy Brassica vegetable pak choi (Brassica rapa ssp. chinensis) as suitable tool to elicit very high concentrations of 1-methoxy-indol3-ylmethyl GS by application of methyl jasmonate (MeJA) [28] the identification of genes involved in this process was possible by comparing expression pattern in pak choi using the $2 \times 104 \mathrm{~K}$ format Brassica Array with publicly available gene expression data from the Arabidopsis AtGenExpress effort [29]. With the functional characterization of the identified genes new genetic tools for breeding healthy vegetables with improved GS composition will be possible in the near future.

\section{Results and discussion}

Increased indole GS biosynthesis in pak choi treated with methyl jasmonate

In a previous study it was shown that different cultivars of the leafy vegetable pak choi (Brassica rapa ssp. chinensis) contain a certain amount of indole GS in their green leaf tissue [30]. The different cultivars can be classified in distinct groups depending on their GS profiles, which are partly linked to the expression of specific genes involved in the aliphatic GS biosynthetic pathway. In a related study it was further demonstrated that a small set of elicitors known to induce GS biosynthesis in various organism is also functional in pak choi [28]. Amongst others it was namely methyl jasmonate (MeJA) that led to an increase of indole GS biosynthesis. In order to further characterize this induction of GS biosynthesis in pak choi seedlings in more detail a concentration series ranging from $100 \mu \mathrm{M}$ to $3 \mathrm{mM}$ was applied and GS accumulation was measured 48 hours after application (Additional file 1: Table S1). As shown in Figure 2A a doubling of specific aliphatic GS could be achieved when applying concentrations of more than $750 \mu \mathrm{M}$ MeJA, and also the amount of the aromatic 2-phenylethyl GS was increased up to 3 fold at such high concentrations applied. As expected, indole GS accumulation was more sensitive to the MeJA application, and the indole GS level was elevated even when the lowest concentration of $100 \mu \mathrm{M}$ was used (Figure 2B). With the application of higher concentrations of MeJA up to $2 \mathrm{mM}$ a further increase of indole GS levels could be achieved until no additional elevation was detected. Notably it was mainly the 1-methoxy-indol-3-ylmethyl GS that was increased up to 30fold in pak choi seedlings after treatment with MeJA.

It is known for a long time that jasmonate, ethylene and salicylic acid upregulate the expression of scores of defense-related genes [31], and our knowledge of the complex network of jasmonate signaling in stress responses and development including hormone cross-talk is continuously increasing [32,33]. With respect to plant resistance GS present classical examples of compounds affecting insect-plant interactions [17] in which the GSmyrosinase defence system is also evolutionary and ecological modulated [19]. In terms of plants defense against pathogens it is further suggested that tryptophan-derived metabolites may act as active antifungal compounds $[9,34]$. Against this background the induced GS biosynthesis was strongly expected in pak choi after treatment with MeJA.

\section{Specific induction of 1-methoxy-indol-3-ylmethyl GS in pak choi seedlings}

In order to analyze the specificity of the increased indole GS biosynthesis in more detail a similar experiment with Arabidopsis seedlings was performed using MeJA concentrations ranging from $200 \mu \mathrm{M}$ up to $5 \mathrm{mM}$. As evident from Figure 3 MeJA application also increased indole GS content in Arabidopsis (Additional file 1: Table S2). However, the increase was much lower in this plant species, and the major elevation was found in the non-methoxylated indol-3-ylmethyl GS. Further experiments demonstrated that pak choi seedlings exert stronger rise of indole GS levels upon MeJA application than adult plants [28], while in Arabidopsis no differences in the elevation between seedlings and adult rosette leaves were detectable (data not shown). This comparison with Arabidopsis thaliana Col-0 ecotype clearly revealed that 

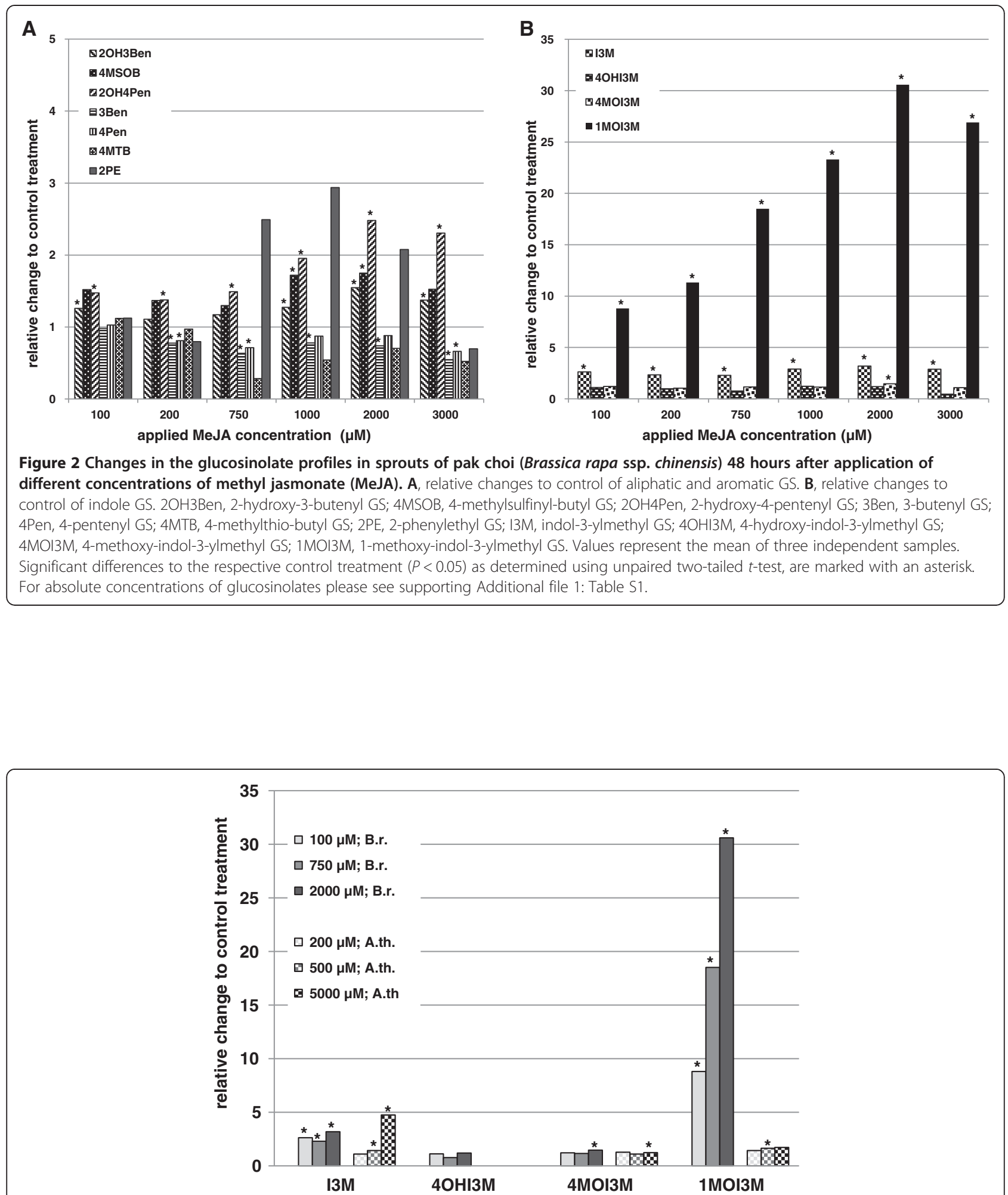

Figure 3 Changes in the indole glucosinolate profiles of $\mathbf{1 2}$ day old seedlings. Pak choi (Brassica rapa ssp. chinensis) (B.r.) and Arabidopsis thaliana Col-0 (A.th.) seedlings were treated with different concentrations of MeJA as indicated and glucosinolate profiles were determined 48 hours after application. B.r. treatment data are the same as in Figure 2; 13M, indol-3-ylmethyl GS; 4OHI3M, 4-hydroxy-indol-3-ylmethyl GS; 4MOI3M, 4-methoxy-indol-3-ylmethyl GS; 1MOI3M, 1-methoxy-indol-3-ylmethyl GS. 4OHI4M was undetectable in A.th. seedlings. Values represent the mean of three independent samples. Significant differences to the respective control treatment $(P<0.05)$ as determined using unpaired two-tailed $t$-test, are marked with an asterisk. For absolute concentrations of glucosinolates please see supporting Additional file 1: Table S2. 
a very strong raise of 1-methoxy-indol-3-ylmethyl GS is specific to pak choi. The unambiguous difference between seedlings of pak choi and Arabidopsis discovered in this glucosinolate profiling analyses was used in further experiments to identify related genes involved in 1methoxy-indol-3-ylmethyl GS biosynthesis of Brassica rapa ssp. chinensis.

\section{Identification of candidate genes using gene expression analysis with the Brassica microarray}

As strong induction of 1-methoxy-indol-3-ylmethyl GS was found 48 hours after application of $2 \mathrm{mM}$ MeJA to pak choi seedlings gene expression differences to control treatments were analyzed in these samples using the Brassica microarray. In order to get maximum amount of information the $2 \times 104 \mathrm{~K}$ array was chosen in the investigation. The elements on the Brassica array were identified by their homology to known genes of Arabidopsis thaliana and were classified to respective bins using MapMan [35] and Mercator [36]. As expected when MeJA was applied to plant seedlings, defense related genes showed the most significantly changed transcript levels (Table 1). With respect to a putative function in GS metabolism [37] the genes with highest expression differences are listed in Table 2. Mainly the transcripts of genes putatively involved in GS degradation were induced, but also genes involved in indole GS core structure formation were strongly elevated and among the most significantly changed. The increased expression of genes specifically involved in indole GS core structure biosynthesis reflects the elevation of indole GS levels. Among the most significantly altered transcripts candidates were selected that are putatively involved in side chain modification of indole GS biosynthesis, namely those that show typical structures of the large gene families of cytochrome P450 monooxygenases or O-methyltransferases (Table 2).

These selected candidates were further evaluated regarding respective expression differences of the related homologs in available Arabidopsis thaliana microarray hybridization experiments using the Genevestigator database [38]. As shown in Table 3 the Arabidopsis homologs of the selected genes involved in GS metabolism were found responsive to MeJA treatments with log2-ratios being 1 or greater. This is in good agreement with the reported modulation of the GS profile in Arabidopsis by defense signaling pathways [39] and is also reflected in results presented in Figure 3. The Arabidopsis homologs of the selected candidate genes show strong variation in their responsiveness to MeJA. While At3g28740 (CYP81D11) and At5g36220 (CYP81D1) were strongly induced by MeJA application, At4g37410 (CYP81F4), At4g37430 (CYP81F1) and At5g42590 (CYP71A16) were only weakly influenced, while At4g35160 (OMT) and
At1g13080 (CYP71B2) showed unchanged expression. As At1g13080, At5g42590 and At3g28740 were already expected to be involved in other metabolic pathways we concentrate in further experiments on At4g37410 and At4g37430 as genes putatively involved in GS metabolism, and on At4g35160 and At5g36220 without any linked pathway identified so far.

\section{GS profiling in Arabidopsis mutants with knock out of the respective candidate gene homologs}

In order to verify a putative involvement of the selected candidate genes in indole GS biosynthesis respective Arabidopsis knock out mutants were profiled for their GS accumulation. Since there are tissue specific differences in the proportional distribution of individual GS with indole GS being mainly present in either roots or old leaves [40] plants were grown in tissue culture and leaves and roots analyzed separately, or GS profiles of leaves of flowering plants grown in the greenhouse were measured (Additional file 1: Table S3). As evident from Table 4 there is one of the four selected Arabidopsis knock out mutants that did not produce 1-methoxy-indol-3ylmethyl GS in any of the tissues analyzed. This confirms the expectation that the Arabidopsis gene product of At4g37410 (CYP81F4) is needed in leaves and roots to synthesize 1-methoxy-indol-3-ylmethyl GS [12,41]. The absence of a metabolic phenotype on GS level in the selected Arabidopsis mutant with knock out in the selected $O$-methyltransferase (Atomt) further shows that at least in Arabidopsis there are other $O$-methyltransferases present which could contribute to the synthesis of 1-methoxyindol-3-ylmethyl GS in leaves. Consequently, it needs to be analyzed whether the $O$-methyltransferase activity is provided through IGMT5 (At1g76790) an O-methyltransferase family protein that is strongly co-expressed with At4g37410 (CYP81F4) as determined using the ATTED-II coexpression database [42]. In addition, in Arabidopsis there are further members of the $O$-methyltransferase family, IGMT1 (At1g21100), IGMT2 (At1g21120) and IGMT4 (At1g21130), that are coexpressed with At5g57220 (AtCYP81F2). At least in an artificial expression system using Nicotiana benthamiana it has been shown that IGMT1 and IGMT2 can be employed for O-methylation of indole GS [12].

As shown previously there is a certain increase of indole GS biosynthesis in Arabidopsis after application of MeJA (Figure 3). Therefore, the selected knocks out mutants of genes responsive to MeJA treatment (Table 3) were also analyzed after application of this elicitor. While mutants in AtCYP81F1 and AtCYP81D1 showed a comparable increase of indole GS biosynthesis as the treated control plants (Table 5), the mutant in AtCYP81F4 did not accumulate any 1-methoxy-indol-3-ylmethyl GS while an expected increase of the precursor indol-3-ylmethyl GS 
Table 1 Expression differences in pak choi seedlings 48 hours after application of methyl jasmonate

\begin{tabular}{|c|c|c|}
\hline Identifier & Log2-fold change & Comparison with Arabidopsis sequences \\
\hline EV086532 & 8.3972 & No similarity found \\
\hline JCVI_8548 & 8.3041 & Weakly similar to (164) AT1G72290| trypsin and protease inhibitor family protein/Kunitz family protein ${ }^{a}$ \\
\hline JCVI_27659 & 7.9237 & Very weakly similar to (93.2) AT1G72290| trypsin and protease inhibitor family protein/Kunitz family protein ${ }^{a}$ \\
\hline EV175386 & 7.7622 & No similarity found \\
\hline JCVI_16491 & 7.6086 & $\begin{array}{l}\text { Moderately similar to (367) AT3G08860| alanine-glyoxylate aminotransferase, putative/beta-alanine-pyruvate } \\
\text { aminotransferase }\end{array}$ \\
\hline JCVI_3681 & 7.5634 & Weakly similar to (176) AT1G73260| trypsin and protease inhibitor family protein/Kunitz family protein ${ }^{a}$ \\
\hline EV210392 & 7.4930 & No similarity found \\
\hline DW997085 & 7.4796 & Moderately similar to (352) AT5G24420| glucosamine/galactosamine-6-phosphate isomerase-related \\
\hline JCVI_25531 & 7.4141 & $\begin{array}{l}\text { Very weakly similar to (82.0) AT1G75940| ATA27 (A. thaliana anther 27); hydrolase, hydrolyzing O-glycosyl } \\
\text { compounds }\end{array}$ \\
\hline JCVI_3301 & 7.4066 & Moderately similar to (292) AT5G07470| PMSR3 (PEPTIDEMETHIONINE SULFOXIDE REDUCTASE 3) ${ }^{a}$ \\
\hline JCVI_38382 & 7.2248 & Moderately similar to (374) AT1G54040| TASTY, ESP (EPITHIOSPECIFIER PROTEIN) ${ }^{b}$ \\
\hline JCVI_20214 & 6.7805 & 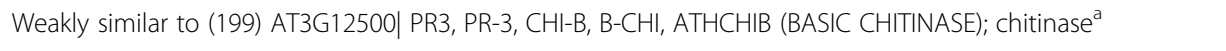 \\
\hline EV022852 & 6.6726 & No similarity found \\
\hline JCVI_19372 & 6.6380 & Moderately similar to (272) AT3G55970| oxidoreductase, 20G-Fe(II) oxygenase family protein \\
\hline JCVI_11797 & 6.5346 & Highly similar to (577) AT2G39310| jacalin lectin family protein ${ }^{a}$ \\
\hline EE568322 & 6.5096 & $\begin{array}{l}\text { Weakly similar to (124) AT3G08860| alanine-glyoxylate aminotransferase, putative/beta-alanine-pyruvate } \\
\text { aminotransferase }\end{array}$ \\
\hline JCVI_2201 & 6.3891 & Weakly similar to (189) AT1G73260| trypsin and protease inhibitor family protein/Kunitz family protein ${ }^{a}$ \\
\hline EX126494 & 6.3312 & Weakly similar to (152) AT1G66700| PXMT1; S-adenosylmethionine-dependent methyltransferase \\
\hline JCVI_19562 & 6.3230 & Weakly similar to (104) AT2G43510| ATTI1 (ARABIDOPSIS THALIANA TRYPSIN INHIBITOR PROTEIN 1) ${ }^{a}$ \\
\hline CD833129 & 6.1070 & Weakly similar to (118) AT1G47540| trypsin inhibitor, putative ${ }^{a}$ \\
\hline EX037239 & 6.1057 & No similarity found \\
\hline JCVI_342 & 6.0609 & Moderately similar to (240) AT1G72290| trypsin and protease inhibitor family protein/Kunitz family protein ${ }^{a}$ \\
\hline EE451932 & 6.0344 & $\begin{array}{l}\text { Very weakly similar to (87.8) AT3G08860| alanine-glyoxylate aminotransferase, putative/beta-alanine-pyruvate } \\
\text { aminotransferase }\end{array}$ \\
\hline JCVI_40366 & 5.9683 & Moderately similar to (435) AT4G03070| AOP1 (2-oxoglutarate dependent dioxygenase 1.1); oxidoreductase \\
\hline JCVI_8581 & 5.9431 & Moderately similar to (349) AT1G52400| BGL1 (BETA-GLUCOSIDASE HOMOLOG 1); hydrolase ${ }^{a}$ \\
\hline JCVI_7526 & 5.9123 & Moderately similar to (442) AT1G52400| BGL1 (BETA-GLUCOSIDASE HOMOLOG 1); hydrolase ${ }^{a}$ \\
\hline JCVI_37097 & 5.7525 & Moderately similar to (309) AT1G66700| PXMT1; S-adenosylmethionine-dependent methyltransferase \\
\hline JCVI_3160 & 5.5469 & Weakly similar to (178) AT4G29270| acid phosphatase class B family protein \\
\hline CX191896 & 5.5195 & No similarity found \\
\hline EX133344 & 5.4854 & Moderately similar to (392) AT1G07440| tropinone reductase, putative/tropine dehydrogenase \\
\hline EX037465 & 5.4674 & Weakly similar to (123) AT3G49360| glucosamine/galactosamine-6-phosphate isomerase family protein \\
\hline JCVI_22700 & 5.4095 & Weakly similar to (196) AT5G59490| haloacid dehalogenase-like hydrolase family protein \\
\hline JCVI_7218 & 5.4086 & $\begin{array}{l}\text { Moderately similar to (291) AT4G37410| CYP81F4 (cytochrome P450, family 81, subfamily F, polypeptide 4); } \\
\text { oxygen binding }\end{array}$ \\
\hline EV124048 & 5.3916 & Weakly similar to (128) AT4G35160| O-methyltransferase family 2 protein ${ }^{\text {b }}$ \\
\hline JCVI_31414 & 5.2952 & Weakly similar to (191) AT4G29710| phosphodiesterase/nucleotide pyrophosphatase-related \\
\hline EV125432 & 5.2734 & $\begin{array}{l}\text { Moderately similar to (240) AT4G37410| CYP81F4 (cytochrome P450, family 81, subfamily F, polypeptide 4); } \\
\text { oxygen binding }\end{array}$ \\
\hline JCVI_33618 & 5.2687 & Moderately similar to (457) AT4G35160| O-methyltransferase family 2 protein ${ }^{b}$ \\
\hline H74959 & 5.2324 & No similarity found \\
\hline JCVI_15025 & 5.2252 & Moderately similar to (311) AT3G12520| SULTR4;2 (sulfate transporter 4;2); sulfate transmembrane transporter \\
\hline EX039068 & 5.1985 & $\begin{array}{l}\text { Weakly similar to (110) AT4G31500| SUR2, RNT1, ATR4, CYP83B1 (CYTOCHROME P450 } \\
\text { MONOOXYGENASE 83B1); oxygen binding }\end{array}$ \\
\hline
\end{tabular}


Table 1 Expression differences in pak choi seedlings 48 hours after application of methyl jasmonate (Continued)

\begin{tabular}{lll}
\hline EX083822 & 5.1636 & Very weakly similar to (91.7) AT1G54040| TASTY, ESP (EPITHIOSPECIFIER PROTEIN) \\
CV432816 & 5.1395 & Moderately similar to (320) AT1G66700| PXMT1; S-adenosylmethionine-dependent methyltransferase \\
JCVI_22851 & 5.1432 & Moderately similar to (255) AT5G06860| PGIP1 (POLYGALACTURONASE INHIBITING PROTEIN 1); protein binding \\
JCVI_7995 & 5.0811 & Moderately similar to (393) AT3G60140| SRG2, DIN2 (DARK INDUCIBLE 2); hydrolase \\
EX117993 & 4.9563 & Moderately similar to (414) AT5G04380| S-adenosyl-L-methionine:carboxyl methyltransferase family protein \\
ES906294 & 4.8431 & Moderately similar to (293) AT1G62660| beta-fructosidase (BFRUCT3)/beta-fructofuranosidase/invertase, vacuolar \\
CV433026 & 4.8167 & Very weakly similar to (80.5) AT3G45140| ATLOX2, LOX2 (LIPOXYGENASE 2) \\
JCVI_19710 & 4.8088 & Moderately similar to (314) AT3G45140| ATLOX2, LOX2 (LIPOXYGENASE 2) \\
EV209435 & 4.7071 & No similarity found \\
JCVI_14756 & 4.7010 & Moderately similar to (319) AT3G08860| alanine-glyoxylate aminotransferase, putative/beta-alanine-pyruvate \\
& & aminotransferase
\end{tabular}

The Brassica $95 \mathrm{~K}$ unigene set was compared to Arabidopsis thaliana TAIR9 genome release and mapped to MapMan bins. Respective Brassica identifiers are shown, and relative changes to controls are given as log2-ratios. Grading of sequence similarity scores of the comparison with Arabidopsis sequences is as follows: highly similar, 501-1000; moderately similar, 201-500; weakly similar, 101-200. a, stress related, MapMan BinCode20; b, sulfur assimilation and glucosinolate metabolism, MapMan.

Table 2 Selected expression differences in pak choi seedlings 48 hours after application of methyl jasmonate

\begin{tabular}{|c|c|c|c|}
\hline & Identifier & Log2-fold change & Comparison with Arabidopsis sequences \\
\hline \multirow{15}{*}{$\begin{array}{l}\text { Glucosinolate } \\
\text { metabolism }\end{array}$} & JCVI_38382 & 7.225 & Moderately similar to At1g54040, ESP, epithiospecifier protein \\
\hline & EX039068 & 5.199 & Weakly similar to At4g31500, SUR2, CYP83B1, chytochrom P450 monooxygenase 83B1 \\
\hline & JCVI_24334 & 4.326 & Highly similar to At2g22330, CYP79B3, cytochrome P450 monooxygenase 79B3 \\
\hline & JCVI_41905 & 4.265 & Moderately similar to At4g39940, AKN2, APS-kinase 2 \\
\hline & JCVI_10889 & 4.238 & Moderately similar to At5g14200, 3-isopropylmalate dehydrogenase \\
\hline & JCVI_10648 & 3.943 & Moderately similar to At4g39940, AKN2, APS-kinase 2 \\
\hline & JCVI_1353 & 3.140 & Moderately similar to At1g54020, myrosinase-associated protein \\
\hline & JCVI_16379 & 3.055 & Highly similar to At4g39950, CYP79B2, cytochrome P450 monooxygenase 79B2 \\
\hline & JCVI_33391 & 2.466 & Highly similar to At4g39950, CYP79B2, cytochrome P450 monooxygenase 79B2 \\
\hline & EV159250 & 2.317 & Weakly similar to At1g52040, MBP1, myrosinase-binding protein 1 \\
\hline & JCVI_2556 & 2.299 & Weakly similar to At1g52030, MBP2, myrosinase-binding protein 2 \\
\hline & JCVI_109 & 2.151 & Moderately similar to At4g31500, SUR2, CYP83B1, chytochrom P450 monooxygenase 83B1 \\
\hline & JCVI_31290 & 2.117 & Moderately similar to At1g24100, UGT74B1 UDP-glucosyl transferase 74B1 \\
\hline & JCVI_15640 & 1.969 & Weakly similar to At1g62540, flavin-containing monooxygenase family protein \\
\hline & JCVI_3890 & 1.953 & Moderately similar to At5g25980, TGG2, glucoside glucohydrolase 2 \\
\hline \multirow{9}{*}{ Candidate genes } & JCVI_7218 & 5.409 & Moderately similar to At4g37410, CYP81F4, cytochrome P450 monooxygenase 81F4 \\
\hline & EV124048 & 5.392 & Weakly similar to At4g35160, O-methyltransferase family protein \\
\hline & EV125432 & 5.273 & Moderately similar to At4g37410, CYP81F4, cytochrome P450 monooxygenase 81F4 \\
\hline & JCVI_33618 & 5.269 & Moderatey similar to At4g35160, O-methyltransferase family 2 protein \\
\hline & JCVI_40877 & 4.207 & Moderately similar to At4g37430, CYP81F1, CYP91A2, cytochrome P450 monooxygenase $81 \mathrm{~F} 1$ \\
\hline & JCVI_39399 & 3.658 & Moderately similar to At1g13080, CYP71B2, cytochrome P450 monooxygenase 71B2 \\
\hline & JCVI_12863 & 3.217 & Weakly similar to At5g42590, CYP71A16, cytochrome P450 monooxygenase 71A16 \\
\hline & EV170929 & 2.549 & Moderately similar to At3g28740, CYP81D11, cytochrome P450 monooxygenase 81D11 \\
\hline & JCVI_8990 & 1.808 & Moderately similar to At5g36220, CYP91A1, CYP81D1, cytochrome P450 monooxygenase \\
\hline
\end{tabular}

The Brassica $95 \mathrm{~K}$ unigene set was compared to Arabidopsis thaliana TAIR9 genome release and mapped to MapMan bins. Respective Brassica identifiers are shown, and relative changes to controls are given as log2-ratios. Grading of sequence similarity scores of the comparison with Arabidopsis sequences is as follows: highly similar, 501-1000; moderately similar, 201-500; weakly similar, 101-200. Genes with significantly altered expression and similarity to Arabidopsis genes with function in GS metabolism and genes with significantly altered expression and similarity to candidate genes of the gene families of cytochrome P450 monooxygenases and O-methyltransferasese are listed. 
Table 3 Evaluation of expression differences upon methyl jasmonate application of Arabidopsis thaliana genes involved in glucosinolate metabolism and respective homologs of candidate genes

\begin{tabular}{|c|c|c|c|}
\hline Arabidopsis gene, encoded protein & Induction & Related pathway & Brassica identifier \\
\hline At1g54040, ESP, epithiospecifier protein & ++ & GS degradation & JCVI_38382 \\
\hline \multirow[t]{2}{*}{ At4g31500, SUR2, CYP83B1, chytochrom P450 monooxygenase 83B1 } & \multirow[t]{2}{*}{++} & \multirow[t]{2}{*}{ GS biosynthesis } & EX039068 \\
\hline & & & JCVI_109 \\
\hline At2g22330, CYP79B3, cytochrome P450 monooxygenase 79B3 & ++ & GS biosynthesis & JCVI_24334 \\
\hline \multirow[t]{2}{*}{ At4g39940, AKN2, APS-kinase 2} & \multirow[t]{2}{*}{++} & \multirow[t]{2}{*}{ Sulfur assimilation } & JCVI_41905 \\
\hline & & & JCVI_10648 \\
\hline At5g14200, 3-isopropylmalate dehydrogenase & ++ & GS biosynthesis & JCVI_10889 \\
\hline At1g54020, myrosinase-associated protein & +++ & GS degradation & JCVI_1353 \\
\hline \multirow[t]{2}{*}{ At4g39950, CYP79B2, cytochrome P450 monooxygenase 79B2 } & \multirow[t]{2}{*}{++} & \multirow[t]{2}{*}{ GS biosynthesis } & JCVI_16379 \\
\hline & & & JCVI_33391 \\
\hline At1g52040, MBP1, myrosinase-binding protein 1 & +++ & GS degradation & EV159250 \\
\hline At1g52030, MBP2, myrosinase-binding protein 2 & +++ & GS degradation & JCVI_2556 \\
\hline At1g24100, UGT74B1 UDP-glucosyl transferase 74B1 & ++ & GS biosynthesis & JCVI_31290 \\
\hline At1g62540, flavin-containing monooxygenase family protein & ++ & GS biosynthesis & JCVI_15640 \\
\hline At5g25980, TGG2, glucoside glucohydrolase 2 & ++ & GS degradation & JCVI_3890 \\
\hline \multicolumn{4}{|l|}{ Homologs of candidate genes, encoded proteins } \\
\hline \multirow[t]{2}{*}{ At4g37410, CYP81F4, cytochrome P450 monooxygenase 81F4 } & \multirow[t]{2}{*}{+} & \multirow[t]{2}{*}{ Putative GS metabolism } & JCVI_7218 \\
\hline & & & EV125432 \\
\hline At4g35160, O-methyltransferase family 2 protein & 0 & Unknown & EV124048 \\
\hline At4g37430, CYP81F1, CYP91A2, cytochrome P450 monooxygenase 81F1 & + & Putative GS metabolism & JCVI_40877 \\
\hline At1g13080, CYP71B2, cytochrome P450 monooxygenase 71B2 & 0 & Putative amino acids and derivatives & JCVI_39399 \\
\hline At5g42590, CYP71A16, cytochrome P450 monooxygenase 71 A16 & + & Put. triterpene, sterole, brassinosteroide & JCVI_12863 \\
\hline At3g28740, CYP81D11, cytochrome P450 monooxygenase 81D11 & +++ & Putative phenylpro-panoid metabolism & EV170929 \\
\hline At5g36220, CYP91A1, CYP81D1, cytochrome P450 monooxygenase & +++ & Unknown & JCVI_8990 \\
\hline
\end{tabular}

The Genevestigator database [37] was used to evaluate expression differences of the selected genes. Arabidopsis genes homologous to the identified MeJA responsive Brassica genes are in the same order as in Table 2. Grading of changes of the log2-ratios is as follows: 0, unchanged with log2-ratio smaller 0.5; +, $\log 2$-ratio between 0.5 and $1 ;++, \log 2$-ratio between 1 and $2.5 ;+++$, log2-ratio larger than 2.5 .

could be observed 48 hours after MeJA application in this mutant. This finally confirms that the gene product of At4g37410, the cytochrome P450 monooxygenase 81F4 is utterly necessary to synthesize 1-methoxy-indole-3ylmethyl GS in Arabidopsis at standard growth conditions.
It additionally demonstrates that there is none of the other P450 monooxygenase $81 \mathrm{~F}$ family proteins involved in 1-methoxy-indole-3-ylmethyl GS synthesis even under conditions of increased biosynthesis when defense related pathways are induced.

Table 4 Glucosinolate content in different tissues of selected Arabidopsis mutants

\begin{tabular}{|c|c|c|c|c|c|c|c|c|c|c|}
\hline Mutant (gene) & Tissue & 3MSOP & 4MSOB & 4MTB & 5MSOP & $8 \mathrm{MSOO}$ & I3M & 4MOI3M & 1MOI3M & Total GS \\
\hline \multirow[t]{2}{*}{ Atcyp81f4 (At4g37410) } & Leaves & $92 \pm 20$ & $90 \pm 14$ & $97 \pm 11$ & $104 \pm 11$ & $102 \pm 19$ & $108 \pm 11$ & $98 \pm 21$ & $-^{*}$ & $95 \pm 11$ \\
\hline & Roots & - & - & - & - & $98 \pm 15$ & $163 \pm 15^{*}$ & $120 \pm 21$ & $-^{*}$ & $113 \pm 13$ \\
\hline \multirow[t]{2}{*}{ Atcyp81f1 (At4g37430) } & Leaves & $99 \pm 3$ & $92 \pm 1$ & $123 \pm 14$ & $102 \pm 4$ & $96 \pm 5$ & $129 \pm 17$ & $99 \pm 6$ & $103 \pm 2$ & $104 \pm 2$ \\
\hline & Roots & - & - & - & - & $93 \pm 1$ & $104 \pm 2$ & $99 \pm 11$ & $79 \pm 15$ & $89 \pm 2$ \\
\hline \multirow[t]{2}{*}{ Atcyp81d1 (At5g36220) } & Leaves & $101 \pm 9$ & $108 \pm 1$ & $81 \pm 4$ & $98 \pm 21$ & $122 \pm 16$ & $87 \pm 4$ & $101 \pm 5$ & $97 \pm 31$ & $96 \pm 2$ \\
\hline & Roots & - & - & - & - & $108 \pm 5$ & $96 \pm 2$ & $101 \pm 7$ & $169 \pm 36$ & $112 \pm 8$ \\
\hline Atomt (At4g35160) & Leaves of flowering plant & $76 \pm 30$ & $103 \pm 43$ & - & - & - & $97 \pm 76$ & $7 \pm 2^{*}$ & $40 \pm 15^{*}$ & $90 \pm 47$ \\
\hline
\end{tabular}

3MSOP, 3-methylsulfinyl-propyl GS; 4MSOB, 4-methylsulfinyl-butyl GS; 4MTB, 4-methylthio-butyl GS; 5MSOP, 5-methylsulfinyl-pentyl GS; 8MSOO, 8-methylsulfinyl-octyl GS; I3M, indol-3-ylmethyl GS; 4MOI3M, 4-methoxy-indol-3-ylmethyl GS; 1MOI3M, 1-methoxy-indol-3-ylmethyl GS. Values represent the mean \pm standard deviation of three to six individual plants homozygous for the respective T-DNA insertion. Significant differences to the respective control tissue $(P<0.05)$ as determined using unpaired two-tailed $t$-test, are marked with an asterisk. Values are given in $\%$ on dry matter basis of the respective control tissue. For absolute concentrations of glucosinolates in the respective control tissue please see supporting Additional file 1: Table S3. -, below detection limit. 
Table 5 Glucosinolate content in Arabidopsis mutants $\mathbf{4 8}$ hours after application of methyl jasmonate

\begin{tabular}{llcccccc}
\hline Mutant & Treatment & Total aliphatic GS & I3M & 4MOI3M & 1MOI3M & Total indol GS & Total GS \\
\hline Atcyp81f4 & $500 \mu \mathrm{M} \mathrm{MeJA}$ & $71 \pm 21$ & $86 \pm 30$ & $162 \pm 12^{*}$ & $-^{*}$ & $87 \pm 23$ & $75 \pm 21$ \\
\hline Atcyp81f1 & $500 \mu \mathrm{M} \mathrm{MeJA}$ & $83 \pm 10$ & $52 \pm 5^{*}$ & $257 \pm 17^{*}$ & $110 \pm 10$ & $81 \pm 5$ & $83 \pm 7$ \\
\hline Atcyp81d1 & $200 \mu \mathrm{M} \mathrm{MeJA}$ & $86 \pm 30$ & $98 \pm 6$ & $72 \pm 6$ & $98 \pm 50$ & $95 \pm 5$ & $86 \pm 26$
\end{tabular}

Total aliphatic GS, 3-methylsulfinyl-propyl GS; 4-methylsulfinyl-butyl GS; 4-methylthio-butyl GS; 5-methylsulfinyl-pentyl GS; 8-methylsulfinyl-octyl GS. Total indol GS, I3M, indol-3-ylmethyl GS; 4MOI3M, 4-methoxy-indol-3-ylmethyl GS; 1MOI3M, 1-methoxy-indol-3-ylmethyl GS. Values represent the mean \pm standard deviation of three individual plants homozygous for the respective T-DNA insertion. Significant differences to the respective control treatment $(P<0.05)$ as determined using unpaired two-tailed $t$-test, are marked with an asterisk. Values are given in $\%$ on dry matter basis of the respective treatment of control plants. -, below detection limit in mutant.

\section{Arabidopsis ecotype Wu-0 without 1-methoxy-indol-3- ylmethyl GS accumulation}

Further evidence of the importance of At4g37410 (CYP81F4) for 1-methoxy-indol-3-ylmethyl GS biosynthesis is coming from a survey of the GS content in leaves and roots of the 19 key accessions [43] used to develop the MAGIC lines [44]. A total of 20 distinct GS could be identified and quantified by Witzel and co-workers, with most of the aliphatic GS showing accession-specific distribution while the indole GS were present in almost all 19 accessions [43] with one exception: ecotype Wu-0 did not contain 1-methoxy-indol-3-ylmethyl GS in any tissue analyzed. Since the corresponding whole genome sequences of all 19 accessions are available [45] the respective sequence variants at locus At4g37410 (http://mus.well. ox.ac.uk/19genomes/variants.tables/) were inspected for the presence of relevant polymorphisms. Indeed, at bp coordinate 18595917 in the pseudo genome and bp coordinate 17592444 of the Col-0 reference genome on chromosome 4 the insertion of one $\mathrm{C}$ nucleotide could be found solely in the accession $\mathrm{Wu}-0$. This insertion produces a frame shift in the coding sequence thus disrupting CYP81F4 and leading to an altered protein sequence from amino acid 390 with a premature stop at amino acid 395. In contrast, the putative functional protein is composed of 501 amino acids in all other accessions that produce 1-methoxy-indole-3-ylmethyl GS. In summary this is an excellent example were publicly available sequence data together with comprehensive metabolite profiling enables the identification of a gene that is putatively involved in the respective metabolic pathway at question. In addition, since the ecotype $\mathrm{Wu}-0$ is an Arabidopsis accession collected from Germany the presence of 1-methoxy-indol-3-ylmethyl GS does not seem to be essential for survival of this ecotype in its natural habitat. As shown previously defense related co-expression networks in Arabidopsis thaliana group together with tryptophan and GS biosynthesis genes in response to stress conditions [16]. Thus, the increase of indole GS biosynthesis in Arabidopsis and the relatively small accumulation of 1-methoxy-indol-3-ylmethyl GS when compared to Brassica rapa ssp. chinensis revealed that this specific indole GS might not play a pivotal role in stress response in Arabidopsis thaliana.

\section{Characterization of the CYP81F4 genes identified in the Brassica rapa genome}

It was already shown that genes involved in the GS biosynthesis exist in more than one copy in the Brassica rapa genome accession Chiifu-401-42 [37]. Besides this there is also a high co-linearity when compared to Arabidopsis thaliana. This co-linearity is similarly found for AtCYP81F4 (At4g37410) surrounded by AtCYP81F3 (At4g37400) and AtCYP81F1 (At4g37430) on Arabidopsis chromosome 4. When compared to Arabidopsis At4g37410 two different orthologues of the Brassica rapa accession Chiifu-401-42 on BAC clones KBrB006J12 and KBrH064I20 could be identified: While KBrB006J12 corresponds to a region on chromosome A01, no match for KBrH064I20 has been found so far. On KBrB006J12 the orthologue to AtCYP81F4 was identified as Bra011759 (BrCYP81F4-1) on the reverse strand on chromosome A01, and is preceded by Bra011758 orthologous to AtCYP81F3 and followed by Bra011761 orthologous to AtCYPF1. On KBrH064I20 the orthologue to AtCYP81F4 was named BrCYP81F4-2, and is preceded by another orthologue to AtCYP81F3 while the following sequence orthologous to AtCYPF1 is corrupted.

In order to analyze the tissue specific expression of the selected genes in more detail isoform specific primer pairs were developed using the respective sequences of the Brassica rapa accession Chiifu-401-42 BAC clones KBrB006J12 and KBrH064I20. Semi-quantitative realtime RT-PCR analysis was performed with cDNA synthesized from RNA isolated from 12 days old seedlings, and leaves and roots of six weeks old Brassica rapa ssp. chinensis plants. As evident from Table 6 expression of all selected genes could be detected in pak choi. In most cases a higher expression was found in leaves than in seedlings and only BrCYP81F4-1 is expressed at a higher level in roots than in leaves. The highest expression level in leaves was detected for BrCYP81F4-2 while BrCYP81F4-1 was the main expressed isoform in roots. This already indicates that the BrCYP81F4 isoforms may play an important role on a tissue-specific level and during development at standard growth conditions.

Further expression analysis was performed with different tissues of pak choi treated with $500 \mu \mathrm{M}$ MeJA. Expression analysis confirmed induction of mainly the two 
Table 6 Semi-quantitative realtime RT-PCR analysis of the selected genes in different tissues of pak choi

\begin{tabular}{lcccc}
\hline Abbreviation & Treatment & Seedlings & Leaves & Roots \\
\hline BrCYP81F1 & Control & $-9.2 \pm 0.64$ & $-3.6 \pm 0.68$ & $-11.6 \pm 2.76$ \\
& $500 \mu \mathrm{M} \mathrm{MeJA}$ & $-7.4 \pm 0.18$ & $-4.2 \pm 2.05$ & $-12.1 \pm 1.52$ \\
\hline BrCYP81F2 & Control & $-10.6 \pm 0.37$ & $-6.8 \pm 1.26$ & $-9.1 \pm 0.94$ \\
& $500 \mu \mathrm{M} \mathrm{MeJA}$ & $-7.7 \pm 0.42$ & $-6.9 \pm 1.43$ & $-8.2 \pm 0.75$ \\
\hline BrCYP81F3-1 & Control & $-6.3 \pm 0.23$ & $-3.4 \pm 0.48$ & $-6.5 \pm 0.57$ \\
& $500 \mu \mathrm{M} \mathrm{MeJA}$ & $-5.7 \pm 0.42$ & $-3.2 \pm 0.44$ & $-6.9 \pm 0.45$ \\
\hline BrCYP81F3-2 & Control & $-6.5 \pm 0.20$ & $-6.4 \pm 0.74$ & $-8.9 \pm 0.32$ \\
& $500 \mu \mathrm{M} \mathrm{MeJA}$ & $-5.7 \pm 0.20$ & $-5.2 \pm 1.41$ & $-6.6 \pm 0.41$ \\
\hline BrCYP81F4-1 & Control & $-7.5 \pm 0.61$ & $-3.5 \pm 0.30$ & $-2.1 \pm 0.30$ \\
& $500 \mu \mathrm{M} \mathrm{MeJA}$ & $-2.4 \pm 0.65$ & $0.3 \pm 0.96$ & $1.5 \pm 0.20$ \\
\hline BrCYP81F4-2 & Control & $-8.5 \pm 0.40$ & $-2.3 \pm 0.37$ & $-6.2 \pm 0.60$ \\
& $500 \mu \mathrm{M} \mathrm{MeJA}$ & $-0.7 \pm 0.75$ & $2.3 \pm 0.52$ & $1.4 \pm 0.60$ \\
\hline BrOMT & Control & $-8.3 \pm 0.51$ & $-5.5 \pm 0.81$ & $-6.7 \pm 0.91$ \\
& $500 \mu \mathrm{M}$ MeJA & $-3.0 \pm 0.99$ & nd & nd
\end{tabular}

Each value represents the $\mathrm{Ct}$ value relative to that of Actin and is given as mean \pm standard deviation of four individual samples. Measurements were repeated twice. Methyl jasmonate (MeJA) treatment was done 48 hours before harvest. nd, not determined.

identified BrCYP81F4 genes in Brassica rapa ssp. chinensis seedlings, leaves and roots treated with MeJA (Table 6). Since there was some increased expression also detectable for other isoforms seedlings of pak choi were treated with a series of different concentrations of MeJA and expression differences to control treatment were analyzed for all BrCYP81F (Figure 4). This unequivocally confirms that both $B r C Y P 81 F 4$ isoforms were most responsive to the elicitor treatment while the others did not show comparable sensitivity to this elicitor. Application of $100 \mu \mathrm{M}$ MeJA already elevated the expression of BrCYP81F4-1 and $\mathrm{BrCYP81F4-2}$ 4fold with highest increase of $\mathrm{BrCYP}$ 81F4-2 of more than 64fold after application of $2 \mathrm{mM}$ MeJA. This confirms that the two isoforms of $B r C Y P 81 F 4$ are the candidate genes from Brassica rapa ssp. chinensis that are crucial for 1-methoxy-indol-3-ylmethyl GS biosynthesis.

Jasmonic acid signaling is a central component of inducible plant defense and the expression of jasmonateinduced responses are tightly regulated by the ecological background of the plant [46] and also by the plant species itself. While in Arabidopsis thaliana tryptophan and GS biosynthesis genes respond to stress conditions [16] there is only relatively small accumulation of 1-methoxyindol-3-ylmethyl GS when compared to Brassica rapa ssp. chinensis. The role of this distinct response to the elicitor and differences in accumulation of a specific defense compound will be the subject of future analysis in an ecological context.

\section{Functional identification of BrCYP81F4 isoforms for biosynthesis of 1-methoxy-indol-3-ylmethyl GS}

In order to finally assess BrCYP81F4 isoform function full length cDNAs of both genes were amplified and heterologously expressed in the Arabidopsis thaliana mutant Atcyp81f4, which does not produce 1-methoxy-indol-3ylmethyl GS. Using oligonucleotide primers developed with the Brassica A genome sequence from Brassica rapa accession Chiifu-401-42 [37] two full length cDNA sequences from Brassica rapa ssp. chinensis coding for putative BrCYP81F4 isoforms were amplified. Both sequences show $90.7 \%$ pair-wise identities and code for proteins of 501 amino acids with $93 \%$ similarity. Compared to the Arabidopsis protein similarities of $85.4 \%$ and $90.2 \%$ could be calculated. The sequences of interest (BrCYP81F4-1

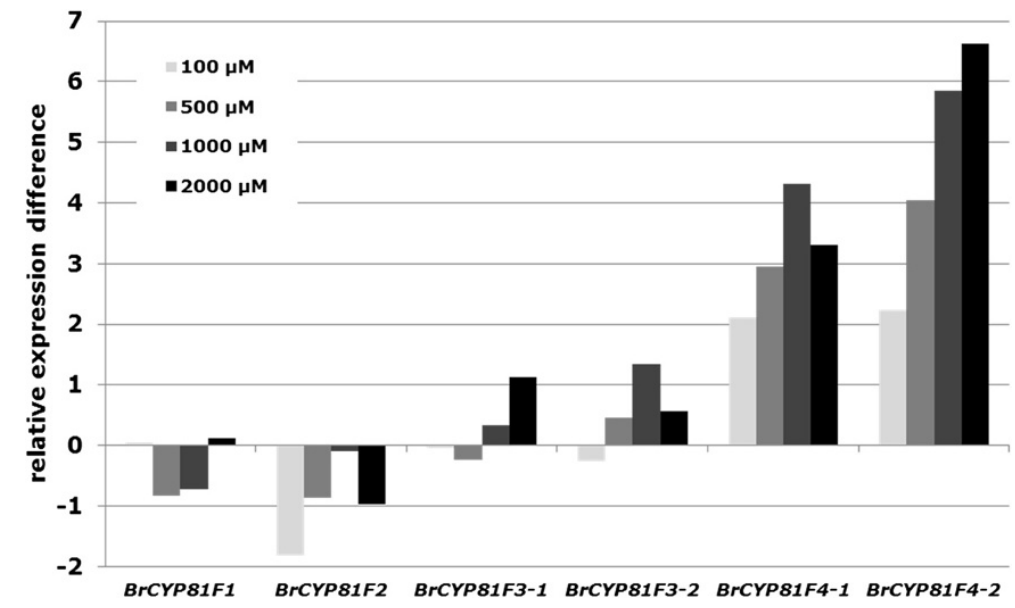

Figure 4 Semi-quantitative realtime RT-PCR analysis of BrCYP81F genes in seedlings of pak choi (Brassica rapa ssp. chinensis) 48 hours after application of different concentrations of methyl jasmonate (MeJA). Values represent the difference of the Ct value relative to that of Actin. Each value represents the mean of nine individual samples. Measurements were repeated twice. Relative expression differences to the control treatment are shown $(\Delta \Delta \mathrm{Ct})$ 
and $B r C Y P 81 F 4-2)$ were recombined into the plant expression vector pK7WG2 [47] and Agrobacterium mediated gene transfer was performed using the knock out mutant Atcyp81f4 as the host. Kanamycin resistant seedlings of the T2 generation were selected and analyzed for heterologous gene expression and GS accumulation. As shown in Table 7 expression of both cDNAs from pak choi in the Atcyp81f4 mutant background led to metabolic complementation with accumulation of 1-methoxy-indol3-ylmethyl GS in leaves and a reduced level of I3M when compared to the mutant without expression of the Brassica rapa ssp. chinensis genes. Although the identical heterologous expression system was used, BrCYP81F4-2 led to much higher accumulation of 1-methoxy-indol-3ylmethyl GS. Whether this difference is caused by a higher protein level of the heterologous enzyme in the mutant plant background or is linked to advanced enzyme activity will be the topic of further studies. Another interesting point here is the significant decrease of I3M in the Atcyp81f4 mutant background when the highly active BrCYP81F4-2 is expressed. In summary the level of indole GS stayed constant in these plants demonstrating unaltered total flux into the indole GS pathway thus indicating no further metabolic regulation by the end products.

\section{Conclusions}

In conclusion this is an explicit example were elicitation of a specific metabolic difference and subsequent comparative microarray analysis together with focused metabolite profiling permits the targeted discovery of genes involved in the respective metabolic pathway. Here this enables the functional attribution of new identified Brassica rapa ssp. chinensis genes to their metabolic role in indole glucosinolate biosynthesis that in the near future will contribute to develop new genetic tools for breeding vegetables with improved glucosinolate profile.

\section{Methods}

Plant material

Seeds of Brassica rapa ssp. chinensis (pak choi) cultivar Black Behi (Allied Botanical, Quezon City, Philippines) were sown on bars of fleece, $3 \mathrm{~g}$ seeds of pak choi, placed in aluminum foil trays $(33 \times 10 \mathrm{~cm})$ filled with perlite. Trays were kept in a greenhouse chamber at $12 \mathrm{~h}$ photoperiod $\left(220 \mu \mathrm{mol} \mathrm{m}{ }^{-2} \mathrm{~s}^{-1}\right.$ of photosynthetic active radiation) and temperature regime of $24 / 20^{\circ} \mathrm{C}$ (day/night) at relative humidity about $75 \%$ for 10 days. The seedlings were watered as needed, no fertilizer was added. To obtain soil grown plants seedlings were germinated and grown on soil at $10 \mathrm{~h}$ photoperiod (photon flux density $150 \mu \mathrm{mol} \mathrm{m}^{-2} \mathrm{~s}^{-1}$, $22^{\circ} \mathrm{C}$ light, $20^{\circ} \mathrm{C}$ dark).

Arabidopsis thaliana L. Heynh Columbia-0 (Col-0), SALK_024438 (Atcyp81f4), SALK_031939 (Atcyp81f1), SALK_005073C (Atcyp81d1), and SALK_053994 (Atomt) were obtained from Nottingham Arabidopsis Stock Centre (University of Nottingham, Loughborough, United Kingdom). Seeds were surface sterilized and aseptically grown on $1 / 2$ strength MS medium including vitamins [48], $0.5 \%$ sucrose and $0.7 \%$ agar. For elicitor treatment $20 \mathrm{mg}$ of Col-0 seeds were spread per petri dish and grown in a greenhouse at $16 \mathrm{~h}$ photoperiod (photon flux density $250 \mu \mathrm{mol} \mathrm{m} \mathrm{m}^{-2} \mathrm{~s}^{-1}$ ) for 10 days. In all other cases seeds were imbibed at $4^{\circ} \mathrm{C}$ darkness $(48 \mathrm{~h})$ and grown in $10 \mathrm{~h}$ photoperiod (photon flux density $150 \mu \mathrm{mol} \mathrm{m} \mathrm{m}^{-2} \mathrm{~s}^{-1}$, $21^{\circ} \mathrm{C}$ ). To obtain soil grown plants seedlings were transferred after three weeks to soil at $10 \mathrm{~h}$ photoperiod (photon flux density $150 \mu \mathrm{mol} \mathrm{m}^{-2} \mathrm{~s}^{-1}, 22^{\circ} \mathrm{C}$ light, $20^{\circ} \mathrm{C}$ dark).

\section{Elicitor treatment}

Methyl jasmonate (Sigma Aldrich, Seelze, Germany) was resolved in water containing $0.01 \%(\mathrm{v} / \mathrm{v})$ Tween 20 to reduce surface tension and water containing $0.01 \%(\mathrm{v} / \mathrm{v})$ Tween 20 was sprayed as control treatment. The 10 days old pak choi seedlings were treated by spraying each bar of fleece with $15 \mathrm{ml}$ of the respective solution. The

Table 7 Glucosinolate profiles in leaves of Arabidopsis mutants Atcyp81f4 transformed with the respective expression vector constructs

\begin{tabular}{lllllllllll}
\hline Mutant lines & $\begin{array}{l}\text { Expression } \\
\text { construct }\end{array}$ & 3MSOP & 4MSOB & 4MTB & 5MSOP & 8MSOO & 4OHI3M & I3M & 4MOI3M & 1MOI3M \\
\hline M3-1 & Control & $0.92 \pm 0.08$ & $6.26 \pm 0.96$ & $0.93 \pm 0.17$ & $0.16 \pm 0.02$ & $0.44 \pm 0.08$ & $0.05 \pm 0.01$ & $1.40 \pm 0.19$ & $0.37 \pm 0.04$ & $0.00 \pm 0.00$ \\
M3-6 & Control & $0.90 \pm 0.22$ & $6.22 \pm 1.54$ & $0.89 \pm 0.37$ & $0.18 \pm 0.05$ & $0.50 \pm 0.14$ & $0.04 \pm 0.01$ & $1.25 \pm 0.23$ & $0.34 \pm 0.04$ & $0.00 \pm 0.00$ \\
\hline M3-1 & 35S::BrCYP81F4-1 & $0.90 \pm 0.18$ & $6.16 \pm 1.54$ & $0.80 \pm 0.11$ & $0.17 \pm 0.04$ & $0.42 \pm 0.12$ & $0.04 \pm 0.01$ & $1.21 \pm 0.16$ & $0.32 \pm 0.04$ & $0.08 \pm 0.01^{*}$ \\
M3-6 & 35S::BrCYP81F4-1 & $0.99 \pm 0.03$ & $7.67 \pm 0.62$ & $0.64 \pm 0.09$ & $0.21 \pm 0.02$ & $0.42 \pm 0.07$ & $0.02 \pm 0.01$ & $1.59 \pm 0.22$ & $0.34 \pm 0.06$ & $0.09 \pm 0.03^{*}$ \\
\hline M3-1 & 35S::BrCYP81F4-2 & $0.86 \pm 0.07$ & $6.47 \pm 1.07$ & $1.01 \pm 0.61$ & $0.19 \pm 0.04$ & $0.66 \pm 0.41$ & $0.00 \pm 0.00^{*}$ & $0.64 \pm 0.35^{*}$ & $0.27 \pm 0.01^{*}$ & $0.97 \pm 0.64^{*}$ \\
M3-6 & 35S::BrCYP81F4-2 & $0.67 \pm 0.13$ & $4.71 \pm 0.90$ & $0.57 \pm 0.05$ & $0.13 \pm 0.03$ & $0.28 \pm 0.06^{*}$ & $0.01 \pm 0.01^{*}$ & $0.43 \pm 0.09^{*}$ & $0.20 \pm 0.04^{*}$ & $0.86 \pm 0.40^{*}$ \\
\end{tabular}

3MSOP, 3-methylsulfinyl-propyl GS; 4MSOB, 4-methylsulfinyl-butyl GS; 4MTB, 4-methylthio-butyl GS; 5MSOP, 5-methylsulfinyl-pentyl GS; 8MSOO, 8-methylsulfinyl-octyl GS; 4OHI3M, 4-hydroxy-indol-3-ylmethyl GS; I3M, indol-3-ylmethyl GS; 4MOI3M, 4-methoxy-indol-3-ylmethyl GS; 1MOI3M, 1-methoxy-indol-3-ylmethyl GS. Values represent the mean \pm standard deviation of three to six individual plants homozygous for the respective T-DNA insertion and transformed with the respective expression constructs. Significant differences to respective control tissue $(P<0.05)$ as determined using unpaired two-tailed $t$-test, are marked with an asterisk. Values are given in $\mu \mathrm{mol}^{*} \mathrm{~g}^{-1}$ dry weight. Control, vector control pK7WG2; 35S::BrCYP81F4-1, expression construct using destination vector pK7WG2 recombined with BrCYP81F4-1; 35S:: BrCYP81F4-2, expression construct using destination vector pK7WG2 recombined with BrCYP81F4-2. 
10 days old Arabidopsis seedlings were treated by spraying each petri dish with $2 \mathrm{ml}$ of the respective solution. 48 hours after treatment the total aerial tissue was harvested. Samples were quickly frozen in liquid nitrogen, subsequently lyophilized, and blended to a fine powder. For each treatment, at least three samples were taken as replicates.

\section{Sample preparation and desulfo-glucosinolate analysis by HPLC}

Glucosinolate concentration was determined as desulfoglucosinolates according to Wiesner et al. [30]. Briefly, $20 \mathrm{mg}$ of powdered samples were extracted and analyzed by HPLC using a Merck HPLC system (Merck-Hitachi, Darmstadt, Germany) with a Spherisorb ODS2 column (Bischoff, Leonberg Germany; particle size $5 \mu \mathrm{m}$, $250 \mathrm{~mm} \times 4 \mathrm{~mm}$ ). Desulfo-glucosinolates were identified based on comparison of retention times and UV absorption spectra with those of known standards. Glucosinolate concentration was calculated by the peak area relative to the area of the internal standard. Each replicate sample was measured in duplicate. Results are given as $\mu \mathrm{mol} \mathrm{g}{ }^{-1}$ dry weight.

\section{Microarray analysis}

The microarray analysis was performed as described [18]. Briefly, frozen pak choi sprout material was ground in liquid nitrogen in an orbital ball mill for $2 \mathrm{~min}$ at a frequency of $30 \mathrm{~Hz} \mathrm{~s}^{-1}$ (MM400 Retsch GmbH, Haan, Germany). Total RNA was extracted using the RNeasy Plant Mini Kit (Qiagen GmbH, Hilden, Germany), including the on-column DNase digestion step with the RNase-free DNase Set (Qiagen). The microarray analysis was done with $1 \mathrm{mg}$ of total RNA isolated from each of three replicates of methyl jasmonate treated and control treated seedlings. Agilent One-Color Gene Expression Microarray analysis following the recommendation of MIAME (http://www.mged.org) was performed at Beckman Coulter Genomics (Morrisville, NC, United States, http://www.beckmangenomics.com/) using the $2 \times 104 \mathrm{k}$ format Brassica Array [49]; http://brassica.bbsrc.ac.uk/). Microarray data are available in the ArrayExpress database (www.ebi.ac.uk/arrayexpress) under accession number E-MTAB-2386. The Open Source Microarray Processing Software Robin (http://mapman.gabipd.org/web/guest/ home) was used to evaluate and calculate results of the log fold change of expression in MeJA treated seedlings in relation to the control [35]. The assignment of the different genes was done by comparison of the translated protein sequences of the $95 \mathrm{k}$ Brassica unigene set with the Arabidopsis TAIR9 database using the Mercator pipeline for automated sequence annotation [36] (http://mapman. gabipd.org/web/guest/app/mercator). For each identifier the gene with the highest homology was provided with identifier and description. The respective bitscores were classified as follows: very weakly similar (bitscore smaller than 100); weakly similar (bitscore 101-200); moderately similar (bitscore 201-500); highly similar (bitscore greater than 500).

\section{Isolation of mutants}

Plants were obtained from the Salk collection [50]. Screening and selection within mutant populations was done following the Signal Salk instructions (http://signal. salk.edu). Genomic DNA was isolated by a standard procedure using NucleoSpin PlantII (Macherey-Nagel $\mathrm{GmbH}$ \& Co. KG, Dueren, Germany). PCR genotyping was performed using the T-DNA LB-specific primer SALK LBb 5'-GCGTGGACCGCTTGCTGCAACT and the gene-specific primer pairs of Atcyp $81 \mathrm{f} 412$ 5' - AGG

\section{Table 8 Oligonucleotide primers used for gene expression analysis}

\begin{tabular}{|c|c|c|}
\hline $\begin{array}{l}\text { Oligonucleotide } \\
\text { abbreviation }\end{array}$ & Sequence & $\begin{array}{l}\text { Accession (Gene } \\
\text { abbreviation) }\end{array}$ \\
\hline At-ACT2f & TCCCTCAGCACATTCCAGCAGAT & At3g18780 \\
\hline At-ACT2r & AACGATTCCTGGACCTGCCTCATC & (AtACT2) \\
\hline At-CYP81F1f & TACTGAGAAATCCAGAAGTACT & At4g37430 \\
\hline At-CYP81F1r & GTTTGGAGGTAAGGAAGCAC & (AtCYP81F1) \\
\hline At-CYP81F4f & TTGTTGAACCACCCAAAAGTTT & At4g37410 \\
\hline At-CYP81F4r & GGAGGTAAGGAAGGTTTGCT & (AtCYP81F4) \\
\hline At-MT2f & CCGGCTTGCGACGCCATTT & At4g35160 \\
\hline At-MT2r & TITATTCTCTCCGATCACCGAT & (AtOMT) \\
\hline At-CYP81D1f & TGCTTAACCATCCTGACGTAA & At5g36220 \\
\hline At-CYP81D1r & CTITAGATATGGTAGCTCGCTA & AtCYP81D1) \\
\hline BrAf & ACGTGGACATCAGGAAGGAC & AC189447 \\
\hline $\mathrm{BrBr}$ & CTTGGTGCAAGTGCTGTGAT & (BrACT2) \\
\hline BrCYP81F1f & TCCCTCGCACGCCGACG & $\begin{array}{l}\text { KBrB006J12.9 } \\
\text { Bra011761 }\end{array}$ \\
\hline BrCYP81F1r & AGGATGCGGCAGCGAGTTA & (BrCYP81F1) \\
\hline BrCYP81F2f & TCTCCTTCTGAAGATCTCAAAA & $\begin{array}{l}\text { KBrB027E01.6 } \\
\text { Bra006830 }\end{array}$ \\
\hline BrCYP81F2r & GTGTTCGCTGCTTCTIITTCT & (BrCYP81F2) \\
\hline BrCYP81F3f1 & GCCGAGATCACCGATGGAA & $\begin{array}{l}\text { KBrB006J12.6 } \\
\text { Bra011758 }\end{array}$ \\
\hline BrCYP81F3r1 & TGAACGTCTTCTCCTCCGC & (BrCYP81F3-1) \\
\hline BrCYP81F3f2 & GCCAAGATCGACGACAGAC & KBrH064|20.2 \\
\hline BrCYP81F3r2 & GTCTTCTCCTCCTTCTCCGA & (BrCYP81F3-2) \\
\hline BrCYP81F4f1 & TTAACGGAAGAGGACATCAAAG & $\begin{array}{l}\text { KBrB006J12.7 } \\
\text { Bra011759 }\end{array}$ \\
\hline BrCYP81F4r1 & AAAGAGGGGAAGGAGACAAAGA & (BrCYP81F4-1) \\
\hline BrCYP81F4f2 & TTAACAGTAGAGGACATCAAGA & KBrH064I20.1 \\
\hline BrCYP81F4r2 & TGGAGGAGAAGGAGAAAAGGA & (BrCYP81F4-2) \\
\hline BrOMTf1 & GGCTGTACCGGAGAGACGA & Bra017700 \\
\hline BrOMTr1 & GCCGTTCTCATCAAGTGGGTG & (BrOMT) \\
\hline
\end{tabular}


GTATTCGTTTTGGAGCA, Atcyp81f4r2 5' - CTTCTC CACCGTTGAACCTC; Atcyp81f112 5' - CTCCAACGA AAGCAACGATT, Atcyp81f1r2 5' - CGAGCATCATCG ACTTCACA; Atcyp81d11 5' - TGCCCATTCTAGAGT GACTGC, Atcyp81d1r 5' - AGAATGATGACCGGAA AACG; Atomtl 5' - CAAGTATTCCCATCGTCTCTCC, Atomtr 5' - ATTGAAAACCATCCTTCGTCAC. Homozygous mutants were isolated from selfed populations of the respective mutant. Gene knock-out was proven by semi-quantitative realtime RT-PCR.

\section{Gene expression analysis by semi-quantitative realtime RT-PCR}

RNA was extracted from $100 \mathrm{mg}$ tissue using the NucleoSpin Plant Kit (Macherey-Nagel GmbH and Co KG), including on-column DNasel digestion. RNA was quantified spectrophotometrically at $260 \mathrm{~nm}$ (Nanodrop ND1000, Technology Inc., USA), and quality was checked using the ratio of absorption at 260 and $280 \mathrm{~nm}$ with a ratio between 1.9 and 2.1 as acceptable. Single-stranded cDNA synthesis was carried out with total RNA using SuperScript ${ }^{\mathrm{mt}}$ III RNaseH-reverse transcriptase (Invitrogen, Life Technologies $\mathrm{GmbH}$, Darmstadt, Germany) with oligo d( $\left.\mathrm{T}_{12-18}\right)$ primers according to the manufacturer's instructions. PCR amplified sequences generated with these oligonucleotide primer pairs and cDNA from pak choi leaves as template were subcloned and verified by sequence analysis. Semiquantitative two-step RT-PCR was performed using a SYBR $^{\circ}$ Green 1 protocol in 96-well reaction plates on an Applied Biosystems 7500 Realtime PCR System. The following thermal profile was used for all reactions: $50^{\circ} \mathrm{C}$ for $2 \mathrm{~min}, 95^{\circ} \mathrm{C}$ for $10 \mathrm{~min}, 40$ cycles of $95^{\circ} \mathrm{C}$ for $30 \mathrm{~s}$ and $60^{\circ} \mathrm{C}$ for $1 \mathrm{~min}$, followed by dsDNA melting curve analysis to ensure amplicon specificity. Each reaction was done in a $10 \mu \mathrm{l}$ volume containing $200 \mathrm{nM}$ of each primer, $3 \mu \mathrm{l}$ of cDNA (1:50) and $7 \mu \mathrm{l}$ of Power SYBR Green Master Mix (Applied Biosystems, Life Technologies, Carlsbad, CA, USA). Data generated by semi-quantitative real-time PCR were collected and compiled using 7500 v2.0.1 software (Applied Biosystems). Data were exported to LinReg software [51] to determine the PCR amplification efficiency for each primer pair. Relative transcript levels of Arabidopsis thaliana were normalized on the basis of expression of At3g18780 (ACT2), and relative transcript levels of Brassica rapa were normalized on the basis of expression of an invariant control orthologous to At3g18780 on $\mathrm{KBrB071H} 12$ by calculating $\Delta C t$, the difference between control and target products $\left(\Delta C t=C t_{\text {GENE }}-C t_{\mathrm{ACT}}\right)$ [52]. Semi-quantitative PCR was performed on at least three biological replicates measured in duplicates for each gene, and non-template controls were included. Gene-specific primer sets are listed in Table 8.

\section{Cloning procedures and plant transformation}

All constructs have been made using a combination of $\mathrm{TOPO}^{\circ}$ and GATEWAY $^{\circ}$ cloning system (Invitrogen). Brassica rapa subsp. chinensis sequences coding for the two identified, putative CYP81F4 were amplified using the Advantage 2 PCR Kit (Clontech, Takara Bio Company, Kyoto, Japan) and primer pairs BrF4-1fg 5' - CACCA TGTTCTACTATGTGATACTCCCT and BrF4-1ro 5' - A ACCTTTGAGTCGGTAACAA; as well as BrF4-2fg 5' - C ACCATGTTTTACTATGTGATTCTCCCT and BrF4-2ro 5' - AACTTTTGACTCGGTAAGAA. PCR products were inserted into the entry vector $\mathrm{pENTR}^{\mathrm{mm}} / \mathrm{SD} / \mathrm{D}-\mathrm{TOPO}^{\circ}$ (Invitrogen), and verified by sequencing (LGC Genomics $\mathrm{GmbH}$, Berlin, Germany). Both sequences of interest (BrCYP81F4-1 (Accession KF612589) and BrCYP81F4-2 (Accession KF612590)) were then recombined into the appropriate destination vector pK7WG2 [47] using GATEWAY $^{\bullet}$ LR Clonase $^{\mathrm{Tw}}$ II enzyme mix according to the manufactures instructions (Invitrogen). Agrobacterium mediated gene transfer was performed according to [53] using two homozygous lines (M3-1, M3-6) of the knock out mutant Atcyp81f4 as the host. Kanamycin resistant seedlings of the $\mathrm{T} 1$ generation were selected and expression of the respective transgene was recorded by semi-quantitative realtime RT-PCR.

\section{Additional file}

Additional file 1: Individual glucosinolate content.

Competing interests

The authors declare that they have no competing interests.

\section{Authors' contributions}

RZ and MS designed the study, MW carried out the elicitor treatments and the metabolite and molecular analyses, RZ carried out the molecular and genetic studies, RZ wrote the manuscript. All authors read and approved the final manuscript.

\section{Acknowledgements}

We gratefully acknowledge the excellent technical assistance from Andrea Maikath and Andrea Jankowsky.

Received: 20 December 2013 Accepted: 24 April 2014 Published: 8 May 2014

\section{References}

1. Mithen R: Glucosinolates - biochemistry, genetics and biological activity. Plant Growth Regul 2001, 34:91-103.

2. Mithen R, Bennett R, Marquez J: Glucosinolate biochemical diversity and innovation in the Brassicales. Phytochemistry 2010, 71:2074-2086.

3. Fahey JW, Zalcmann AT, Talalay P: The chemical diversity and distribution of glucosinolates and isothiocyanates among plants. Phytochemistry 2001, 56:5-51

4. Clarke DB: Glucosinolates, structures and analysis in food. Anal Methods 2010, 2:310-325.

5. Kliebenstein DJ, Kroymann J, Brown P, Figuth A, Pedersen D, Gershenzon J, Mitchell-Olds T: Genetic control of natural variation in Arabidopsis glucosinolate accumulation. Plant Physiol 2001, 126:811-825.

6. Grubb CD, Abel S: Glucosinolate metabolism and its control. Trends Plant Sci 2006, 11:89-100. 
7. Halkier BA, Gershenzon J: Biology and biochemistry of glucosinolates. Annu Rev Plant Biol 2006, 57:303-333.

8. Sonderby IE, Geu-Flores F, Halkier BA: Biosynthesis of glucosinolates - gene discovery and beyond. Trends Plant Sci 2010, 15:283-290.

9. Clay NK, Adio AM, Denoux C, Jander G, Ausubel FM: Glucosinolate metabolites required for an Arabidopsis innate immune response. Science 2009, 323:95-101.

10. Bednarek P, Pislewska-Bednarek M, Svatos A, Schneider B, Doubsky J, Mansurova M, Humphry M, Consonni C, Panstruga R, Sanchez-Vallet A, Molina A, Schulze-Lefert P: A glucosinolate metabolism pathway in living plant cells mediates broad spectrum antifungal defense. Science 2009, 323:101-106.

11. Pfalz M, Vogel H, Kroymann J: The gene controlling the indole glucosinolate modifier1 quantitative trait locus alters indole glucosinolate structures and aphid resistance in Arabidopsis. Plant Cell 2009, 21:985-999.

12. Pfalz M, Mikkelsen MD, Bednarek P, Olsen CE, Halkier BA, Kroymann J: Metabolic engineering in Nicotiana benthamiana reveals key enzyme functions in Arabidopsis indole glucosinolate modification. Plant Cell 2011, 23:716-729.

13. Burow $M$, Wittstock U: Regulation and function of specifier proteins in plants. Phytochem Rev 2009, 8:87-99.

14. Zang YX, Kim HU, Kim JA, Lim MH, Jin M, Lee SC, Kwon SJ, Lee SI, Hong JK, Park TH, Mun JH, Seol YJ, Hong SB, Park BS: Genome-wide identification of glucosinolate synthesis genes in Brassica rapa. FEBS J 2009, 276:3559-3574.

15. Gigolashvili T, Yatusevich R, Berger B, Muller C, Flugge UI: The R2R3-MYB transcription factor HAG1/MYB28 is a regulator of methionine-derived glucosinolate biosynthesis in Arabidopsis thaliana. Plant J 2007, 51:247-261.

16. Gigolashvili T, Berger B, Mock HP, Muller C, Weisshaar B, Flugge UI: The transcription factor HIG1/MYB51 regulates indolic glucosinolate biosynthesis in Arabidopsis thaliana. Plant J 2007, 50:886-901.

17. Van Hopkins RJ, Dam MN, Van Loon JJA: Role of glucosinolates in insect-plant relationships and multitrophic interactions. Annu Rev Entomol 2009, 54:57-83.

18. Mewis I, Schreiner M, Nguyen CN, Krumbein A, Ulrichs C, Lohse M, Zrenner R: UV-B irradiation changes specifically the secondary metabolite profile in broccoli sprouts: induced signaling overlaps with defense response to biotic stressors. Plant Cell Physiol 2012, 53:1546-1560.

19. Kliebenstein DJ, Kroymann J, Mitchell-Olds T: The glucosinolate-myrosinase system in an ecological and evolutionary context. Curr Opin Plant Biol 2005, 8:264-271

20. Dixon GR: Vegetable Brassicas and Related Crucifers. Wallingford, UK: CAB International; 2007

21. Verkerk R, Schreiner M, Krumbein A, Ciska E, Holst B, Rowland I, De Schrijver R, Hansen M, Gerhauser C, Mithen R, Dekker M: Glucosinolates in Brassica vegetables: the influence of the food supply chain on intake, bioavailability and human health. Mol Nutr Food Res 2009, 53:Issue Supplement 2 S219.

22. Engel E, Baty C, Le Corre D, Souchon I, Martin N: Flavor-active compounds potentially implicated in cooked cauliflower acceptance. J Agric Food Chem 2002, 50:6459-6467.

23. Fahey JW, Zhang YS, Talalay P: Broccoli sprouts: an exceptionally rich source of inducers of enzymes that protect against chemical carcinogens. Proc Natl Acad Sci U S A 1997, 94:10367-10372.

24. Mithen R, Faulkner K, Magrath R, Rose P, Williamson G, Marquez J: Development of isothiocyanate-enriched broccoli, and its enhanced ability to induce phase 2 detoxification enzymes in mammalian cells. Theor Appl Genet 2003, 106:727-734.

25. Kassie F, Parzefall W, Musk S, Johnson I, Lamprecht G, Sontag G, Knassmuller S: Genotoxic effects of crude juices from Brassica vegetables and juices and extracts from phytopharmaceutical preparations and spices of cruciferous plants origin in bacterial and mammalian cells. Chem Biol Interact 1996, 102:1-16.

26. Baasanjav-Gerber $C$, Monien BH, Mewis I, Schreiner M, Barillari J, lori R, Glatt H: Identification of glucosinolate congeners able to form DNA adducts and to induce mutations upon activation by myrosinase. Mol Nutr Food Res 2011, 55:783-792.

27. Glatt H, Baasanjav-Gerber C, Schumacher F, Monien BH, Schreiner M, Frank H, Seidel A, Engst W: 1-Methoxy-3-indolylmethyl glucosinolate; a potent genotoxicant in bacterial and mammalian cells: Mechanisms of bioactivation. Chem Biol Interact 2011, 192:81-86.
28. Wiesner $M$, Hanschen $F S$, Schreiner M, Glatt $H$, Zrenner R: Elicitor induced production of 1-methoxy-indol-3-ylmethyl glucosinolate by jasmonic acid and methyl jasmonate in sprouts and leaves of pak choi (Brassica rapa ssp. chinensis). Int J Mol Sci 2013, 14:14996-15016.

29. Schmid M, Davison TS, Henz SR, Pape UJ, Demar M, Vingron M, Schölkopf B, Weigel D, Lohmann JU: A gene expression map of Arabidopsis thaliana development. Nat Genet 2005, 37:501-506.

30. Wiesner M, Zrenner R, Krumbein A, Glatt H, Schreiner M: Genotypic variation of the glucosinolate profile in pak choi (Brassica rapa ssp. chinensis). J Agric Food Chem 2013, 61:1943-1953.

31. Reymond P, Farmer EE: Jasmonate and salicylate as global signals for defense gene expression. Curr Opin Plant Biol 1998, 1:404-411.

32. Wasternack C: Jasmonates: an update on biosynthesis, signal transduction and action in plant stress response, growth and development. Ann Bot 2007, 100:681-697.

33. Wasternack C, Hause B: Jasmonates: biosynthesis, perception, signal transduction and action in plant stress response, growth and development. An update to the 2007 review in Annals of Botany. Ann Bot 2013, 111:1021-1058.

34. Iven T, König S, Singh S, Braus-Strohmeyer SA, Bischoff M, Tietze LF, Braus GH, Lipka V, Feussner I, Dröge-Laser W: Transcriptional activation and production of tryptophan-derived secondary metabolites in Arabidopsis roots contribute to the defense against the fungal vascular pathogen Verticillium longisporun. Mol Plant 2012, 5:1389-1402.

35. Lohse M, Nunes-Nesi A, Krueger P, Nagel A, Hannemann J, Giorgi FM, Childs L, Osorio S, Walther D, Selbig J, Sreenivasulu N, Stitt M, Fernie AR, Usadel B: Robin: an intuitive wizard application for R-based expression microarray quality assessment and analysis. Plant Physiol 2010, 153:642-651.

36. Lohse M, Nagel A, Herter T, May P, Schroda M, Zrenner R, Tohge T, Fernie AR, Stitt M, Usadel B: Mercator: a fast and simple web server for genome scale functional annotation of plant sequence data. Plant Cell Environ 2013, doi:10.1111/pce.12231.

37. The Brassica rapa Genome Sequencing Project Consortium, Wang X, Wang $H_{t}$ Wang J, Sun R, Wu J, Liu S, Bai Y, Mun JH, Bancroft I, Cheng F, Huang S, Li X, Hua W, Wang J, Wang X, Freeling M, Pires CJ, Paterson AH, Chalhoub B, Wang B, Hayward A, Sharpe AG, Park BS, Weisshaar B, Liu B, Li B, Liu B, Tong C, Song $C$, et al: The genome of the mesopolyploid crop species Brassica rapa. Nat Genet 2011, 43:1035-1039.

38. Hruz T, Laule O, Szabo G, Wessendorp F, Bleuler S, Oertle L, Widmayer P, Gruissem W, Zimmermann P: Genevestigator v3: a reference expression database for the meta-analysis of transcriptomes. Adv Bioinformatics 2008:420747.

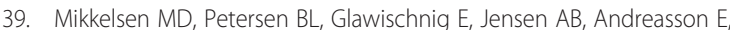
Halkier BA: Modulation of CYP79 genes and glucosinolate profiles in Arabidopsis by defense signaling pathways. Plant Physiol 2003, 131:298-308.

40. Brown PD, Tokuhisa JG, Reichelt M, Gershenzon J: Variation of glucosinolate accumulation among different organs and developmental stages of Arabidopsis thaliana. Phytochemistry 2003, 62:471-481.

41. Kai K, Takahashi H, Saga H, Ogawa T, Kanaya S, Ohta D: Metabolomic characterization of the possible involvement of a Cytochrome P450, CYP81F4, in the biosynthesis of indolic glucosinolate in Arabidopsis. Plant Biotechnol 2011, 28:379-385.

42. Obayashi T, Nishida K, Kasahara K, Kinoshita K: ATTED-II updates: condition-specific gene coexpression to extend coexpression analyses and applications to a broad range of flowering plants. Plant Cell Physi 2011, 52:213-219.

43. Witzel K, Hanschen FS, Schreiner M, Krumbein A, Ruppel S, Grosch R: Verticillium suppression is associated with the glucosinolate composition of Arabidopsis thaliana leaves. PLoS One 2013, 8:e71877.

44. Kover PX, Valdar W, Trakalo J, Scarcelli N, Ehrenreich IM, Purugganan MD, Durrant C, Mott R: A multiparent advanced generation inter-cross to fine-map quantitative traits in Arabidopsis thaliana. PLoS Genet 2009, 5:e1000551.

45. Gan X, Stegle O, Behr J, Steffen JG, Drewe P, Hildebrand KL, Lyngsoe R, Schultheiss SJ, Osborne EJ, Sreedharan VT, Kahles A, Bohnert R, Jean G, Derwent P, Kersey P, Belfield EJ, Harberd NP, Kemen E, Toomajian C, Kover PX, Clark RM, Rätsch G, Mott R: Multiple reference genomes and transcriptomes for Arabidopsis thaliana. Nature 2011, 477:419-423.

46. Ballaré CL: Jasmonate-induced defences: a tale of intelligence, collaborators and rascals. Trends Plant Sci 2011, 16:249-257. 
47. Karimi M, Inze D, Depicke A: GATEWAY vectors for Agrobacterium-mediated plant transformation. Trends Plant Sci 2002, 7:193-195.

48. Murashige T, Skoog F: A revised medium for rapid growth and bio assays with tobacco tissue cultures. Physiol Plant 1962, 15:473-497.

49. Trick M, Cheung F, Drou N, Fraser F, Lobenhofer EK, Hurban PM, Magusin A, Town C, Bancroft I: A newly-developed community microarray resource for transcriptome profiling in Brassica species enables the confirmation of Brassica-specific expressed sequences. BMC Plant Biol 2009, 9:50.

50. Alonso JM, Stepanova AN, Leisse TJ, Kim CJ, Chen H, Shinn P, Stevenson DK, Zimmerman J, Barajas P, Cheuk R, Gadrinab C, Heller C, Jeske A, Koesema E, Meyers CC, Parker H, Prednis L, Ansari Y, Choy N, Deen H, Geralt M,

Hazari N, Horn E, Karnes M, Mulholland C, Ndubaku R, Schmidt I, Guzman P, Aguilar-Henonin L, Schmid M, et al: Genome-wide insertional mutagenesis of Arabidopsis thaliana. Science 2003, 301:653-657.

51. Ruijter J, Ramakers C, Hoogaars W, Karlen Y, Bakker O, van den Hoff M, Moorman A: Amplification efficiency: linking baseline and bias in the analysis of quantitative PCR data. Nucleic Acids Res 2009, 37. doi:10.1093/nar/gkp045.

52. Livak KJ, Schmittgen TD: Analysis of relative gene expression data using real-time quantitative PCR and the 2(-Delta Delta $C(T)$ ) method. Methods 2001, 25:402-408.

53. Clough SJ, Bent AF: Floral dip: a simplified method for Agrobacterium-mediated transformation of Arabidopsis thaliana. Plant $J$ 1998, 16:735-743.

doi:10.1186/1471-2229-14-124

Cite this article as: Wiesner et al:: Functional identification of genes responsible for the biosynthesis of 1-methoxy-indol-3-ylmethylglucosinolate in Brassica rapa ssp. chinensis. BMC Plant Biology 2014 14:124.

\section{Submit your next manuscript to BioMed Central and take full advantage of:}

- Convenient online submission

- Thorough peer review

- No space constraints or color figure charges

- Immediate publication on acceptance

- Inclusion in PubMed, CAS, Scopus and Google Scholar

- Research which is freely available for redistribution 